\title{
Hawking Radiation via Damour-Ruffini Method in Squashed Charged Rotating Kaluza-Klein Black Holes
}

\author{
Ji-Wan Hu${ }^{1} \cdot$ Jing-He Wu ${ }^{2} \cdot$ Xian-Ming Liu ${ }^{1}$
}

Received: 13 September 2016 / Accepted: 9 November 2016 / Published online: 3 December 2016 (C) The Author(s) 2016. This article is published with open access at Springerlink.com

\begin{abstract}
Using the Damour-Ruffini method, Hawking radiation of charged particles from squashed charged rotating five-dimensional Kaluza-Klein black holes is investigated extensively. Under the generalized tortoise coordinate transformation, Hawking temperature of the black holes is calculated by using charged scalar particles and Dirac fermions respectively. We find that the obtained Hawking temperature for charged Dirac fermions is the same as for charged scalar particles. What's more, the spectrum of Hawking radiation contains the information of the size of the extra dimension, which could provide insight for further investigation of large extra dimensions in the future.
\end{abstract}

Keywords Hawking radiation · Black hole · Damour-Ruffini method · Charged particles

\section{Introduction}

Hawking radiation is a significant quantum gravity effect arising in the black hole spacetime with an event horizon. An alternative method of deviating Hawking radiation is proposed by Damour and Ruffini [1] and extended by Sannan in [2], since Hawking's original investigation [3, 4]. This method relies on relativistic quantum field theory in curved space-time background without introducing the second quantization. It only requires the existence of a future horizon and is completely independent of any dynamical details of the process leading to the formation of the horizon. The crucial procedure is to use the tortoise coordinate transformation to acquire the tunneling wave function in the complexified

Xian-Ming Liu

liuxianming1980@163.com

1 Department of Physics, Hubei University for Nationalities, Enshi, 445000, Hubei, China

2 Department of Physics and Electronic Engineering, Henan Institute of Education, Zhengzhou, 450046, China 
manifold of the black hole space-time. Recently,the tunneling mechanism [5-7] has been widely used to discuss Hawking radiation, because it reflects the physical process of particle emission from an event horizon. One approach uses Hamilton's equation on null geodesics to calculate Hawking temperature [5-8], while the other uses Hamilton-Jacobi equation to obtain Hawking temperature [9-12]. Interestingly, it is found that after considering the the emitted particles self-gravitation, the spectrum from black holes is not purely thermal. So the tunneling process is a possible mechanism to explain the information loss paradox.

In recent years, squashed Kaluza-Klein black holes have attracted much attention mainly due to their special topology and asymptotical structure [13-20]. All of them behave as five-dimensional black holes near the horizon but look four-dimensional Minkowski spacetime for an observer at the spatial infinity. In Squashed Kaluza-Klein black holes, the size of compactified extra dimension is characterized by an adjustable parameter $r_{\infty}$. Recent studies have found that in Squashed Kaluza-Klein black holes, some interesting physical phenomena, such as the Hawking radiation and thermodynamics [21-23], the quasinormal frequencies [24-26], the geodesic precession effect [27], the Kerr/CFT correspondence [28] and the strong gravitational lensing [29, 30],contain the information of the size of the extra dimension, which could provide insight for further investigation of large extra dimensions.

Therefore it is of great interest to investigate the quantum effect in five dimensional Kaluza-Klein black holes with compact extra dimensions. In this paper,we are going to use Damour-Ruffini method to further study Hawking radiation from squashed charged rotating five-dimensional black holes. We will consider both charged scalar particles and Dirac fermions.

The plan of our paper is organized as follows. In the next section, we review the squashed charged rotating Kaluza-Klein black holes. In Sections 3 and 4, under the generalized tortoise coordinate transformation, we adopt the Damour-Ruffini method to calculate Hawking temperature by using charged scalar particles and Dirac fermions respectively. The conclusions are given in the last section. We use natural units as $c=G=\hbar=\kappa_{B}=1$ throughout this paper.

\section{The Squashed Charged Rotating Kaluza-Klein Black Holes}

We start with a squashed charged rotating Kaluza-Klein black hole which is a solution of the five-dimensional Einstein-Maxwell theory with a Chern-Simon term. The squashed charged rotating Kaluza-Klein black holes can be obtained by applying the squashing transformation techniques to the non-BPS charged rotating black holes found by Cvetic et al. [31, 32]. The metric and the corresponding gauge potential can be written in the following form as [33]

$$
d s^{2}=-\frac{w(r)}{h(r)} d t^{2}+\frac{k(r)^{2}}{w(r)} d r^{2}+\frac{r^{2}}{4}\left[k(r)\left(\sigma_{1}^{2}+\sigma_{2}^{2}\right)+h(r)\left(f(r) d t+\sigma_{3}\right)^{2}\right],
$$

and

$$
A=\frac{\sqrt{3} q}{2 r^{2}}\left(d t-\frac{a}{2} \sigma_{3}\right)
$$

with left-invariant 1 -forms on $S^{3}$

$$
\begin{aligned}
& \sigma_{1}=\cos \varphi d \theta+\sin \varphi \sin \theta d \phi, \\
& \sigma_{2}=-\sin \varphi d \theta+\cos \varphi \sin \theta d \phi, \\
& \sigma_{3}=d \varphi+\cos \theta d \phi,
\end{aligned}
$$


where $0 \leq \theta \leq \pi, 0 \leq \phi<2 \pi, 0 \leq \varphi<4 \pi$. The metric functions $w(r), h(r), k(r)$ and $f(r)$ are given by

$$
\begin{aligned}
w(r) & =\frac{\left(r^{2}+q\right)^{2}-2(m+q)\left(r^{2}-a^{2}\right)}{r^{4}} \\
& =\frac{\left(r^{2}-r_{+}^{2}\right)\left(r^{2}-r_{-}^{2}\right)}{r^{4}}, \\
h(r) & =1-\frac{a^{2} q^{2}}{r^{6}}+\frac{2 a^{2}(m+q)}{r^{4}}, \\
k(r) & =\frac{\left(r_{\infty}^{2}+q\right)^{2}-2(m+q)\left(r_{\infty}^{2}-a^{2}\right)^{2}}{r_{\infty}^{2}-r^{2}} \\
& =\frac{\left(r_{\infty}^{2}-r_{+}^{2}\right)\left(r_{\infty}^{2}-r_{-}^{2}\right)}{\left(r_{\infty}^{2}-r^{2}\right)^{2}}, \\
f(r) & =-\frac{2 a}{r^{2} h(r)}\left(\frac{2 m+q}{r^{2}}-\frac{q^{2}}{r^{4}}\right) .
\end{aligned}
$$

It is worth noting that the functions $k(r)$, which describes the squashing of the horizons, would give a transformation from asymptotically flat solutions to Kaluza-Klein type solutions [19]. The polar coordinate $r$ is limited in the range $0<r<r_{\infty}$, where the parameter $r_{\infty}$ can be used to adjust the size of compactified extra dimension. When $r_{\infty} \rightarrow \infty$, $k(r)=1$, it leads to the Cvetic solution [31,32].

As discussed in $[19,33]$, in order to remove the coordinate singularity at $r=r_{\infty}$, one can introduce a radial coordinate transformation

$$
\rho=\rho_{0} \frac{r^{2}}{r_{\infty}^{2}-r^{2}}
$$

with

$$
\rho_{0}^{2}=\frac{\left(r_{\infty}^{2}+q\right)^{2}-2(m+q)\left(r_{\infty}^{2}-a^{2}\right)}{4 r_{\infty}^{2}}=\frac{\left(r_{\infty}^{2}-r_{+}^{2}\right)\left(r_{\infty}-r_{-}^{2}\right)}{4 r_{\infty}^{2}}
$$

where the parameter $\rho_{0}$ is a scale of transition from five-dimensional space-time to an effective four-dimensional one. What's more, as has already been pointed out in [23, 33], the asymptotic time differs from the coordinate one and takes the form

$$
\tilde{t}=\frac{\rho_{0}}{l} t=\beta t
$$

where the parameter $l$ characterizes the size of extra dimension, given by

$$
l^{2}=\frac{r_{\infty}^{6}-a^{2}\left(q^{2}-2(m+q) r_{\infty}^{2}\right)}{4 r_{\infty}^{4}}
$$


The parameters $m, a, q$ are relate to the Komar mass, the angular momenta and the electric charge as follows

$$
\begin{aligned}
& M_{\text {Komar }}=\pi l \frac{2 r_{\infty}^{6}\left(m r_{\infty}^{2}-q^{2}\right)-2 a^{4}(m+q) q^{2}-a^{2}\left(q^{4}-4 m q^{2} r_{\infty}^{2}+\left(4 m^{2}+4 m q+3 q^{2}\right) r_{\infty}^{4}\right)}{2 r_{\infty}^{2}\left(r_{\infty}^{6}-a^{2}\left(q^{2}-2(m+q) r_{\infty}^{2}\right)\right) \rho_{0}}, \\
& J_{\phi}=0, \\
& J_{\varphi}=-\pi a l \frac{a^{2} q^{3}+3 q^{2} r_{\infty}^{4}-2(2 m+q) r_{\infty}^{6}}{4 r_{\infty}^{4} \sqrt{r_{\infty}^{6}-a^{2}\left(q^{2}-2(m+q) r_{\infty}^{2}\right)}}, \\
& Q=-\frac{\sqrt{3}}{2} \pi q,
\end{aligned}
$$

where $J_{\phi}, J_{\varphi}$ are associated with the Killing vector fields $\partial_{\phi}$ and $\partial_{\varphi}$ respectively. As the parameters $a$ and $q$ tend to zero, one can find that the metric (1) reduces to that of a fivedimensional Schwarzschild black hole with squashed horizon. The outer and inner horizons are located at $r_{+}$and $r_{-}$, which are given by

$$
r_{ \pm}^{2}=m \pm \sqrt{(m+q)\left(m-q-2 a^{2}\right)},
$$

Thus the shape of the black hole event horizon is deformed by the parameter $k\left(r_{+}\right)$. The surface gravity $\kappa_{\star}$ of the black hole can also be obtained as

$$
\begin{aligned}
\kappa_{\star} & =\frac{r_{+}^{2}-r_{-}^{2}}{r_{\infty}\left(r_{\infty}^{2}-r_{-}^{2}\right)} \sqrt{\frac{r_{\infty}^{2}-r_{+}^{2}}{r_{\infty}^{2}-r_{-}^{2}}} \sqrt{\frac{r_{\infty}^{6}-a^{2} q^{2}+2 a^{2}(m+q) r_{\infty}^{2}}{r_{+}^{6}-a^{2} q^{2}+2 a^{2}(m+q) r_{+}^{2}}}, \\
& =\frac{r_{+}^{2}-r_{-}^{2}}{r_{\infty}^{2}-r_{-}^{2}} \sqrt{\frac{r_{\infty}^{2}-r_{+}^{2}}{r_{\infty}^{2}-r_{-}^{2}}} \frac{2 r_{\infty} l}{\sqrt{r_{+}^{6}-a^{2} q^{2}+2 a^{2}(m+q) r_{+}^{2}}} .
\end{aligned}
$$

It is obvious that $\kappa_{\star}$ is relevant to the size of extra dimension.

\section{Hawking Radiation of the Charged Scalar Particles}

In this section, We will study Hawking radiation of charged scalar particles in the squashed charged rotating Kaluza-Klein black hole using Damour-Ruffini method. The Klein-Gordon equation of a charged scalar particle in a curved space-time is given by

$$
\frac{1}{\sqrt{-g}} \frac{\partial}{\partial x^{\mu}}\left[\sqrt{-g} g^{\mu \nu}\left(\frac{\partial}{\partial x^{\nu}}-i e A_{\nu}\right)\right] \psi-i e A_{\mu}\left[g^{\mu \nu}\left(\frac{\partial}{\partial x^{\nu}}-i e A_{\nu}\right)\right] \psi=\mu_{0}^{2} \psi
$$

where $\mu_{0}$ denotes the mass of the charged scalar particle and $A_{\mu}$ is the five-dimensional electromagnetic potential.

Because $\left(\frac{\partial}{\partial t}\right)^{a},\left(\frac{\partial}{\partial \phi}\right)^{a}$ and $\left(\frac{\partial}{\partial \varphi}\right)^{a}$ are Killing vector fields, we could separate the variables as follows

$$
\psi(t, r, \theta, \phi, \varphi)=e^{-i(E \tilde{t}-J \phi-L \varphi)} R(r) \chi(\theta),
$$


where $E$ is the particles's energy and $J$ and $L$ are the angular momenta corresponding to the angles $\phi$ and $\varphi$, respectively. $\tilde{t}$ denotes the time for a distant observed which is defined by the equation (7). Then we can get the radial equation

$$
\frac{w^{2}(r)}{h(r) k^{2}(r) \beta^{2}} \frac{d^{2}}{d r^{2}} R(r)+B(r) \frac{d}{d r} R(r)+C(r)^{2} R(r)-D(r) R(r)=0,
$$

and the angular equation

$$
4 \frac{d^{2}}{d \theta^{2}} \chi(\theta)+4 \cot \theta \frac{d}{d \theta} \chi(\theta)-\left[4\left(\frac{J}{\sin \theta}-L \cot \theta\right)^{2}-\lambda\right] \chi(\theta)=0
$$

respectively, where

$$
\begin{aligned}
& B(r)=\frac{w^{2}(r)}{h(r) k^{2}(r) \beta^{2}}\left(\frac{3}{r}+\frac{2 k^{\prime}(r)}{k(r)}\right)+\frac{w(r)}{h(r) \beta^{2}} \frac{d}{d r}\left(\frac{w(r)}{k^{2}(r)}\right), \\
& C(r)=E+\frac{f(r) L}{\beta}+\frac{\sqrt{3} q e}{2 \beta r^{2}}\left(1+\frac{a f(r)}{2}\right), \\
& D(r)=\frac{w(r)}{h(r) \beta^{2}} \mu_{0}^{2}+\frac{w(r)}{r^{2} h^{2}(r) \beta^{2}}\left(2 L+\frac{\sqrt{3} q e a}{2 r^{2}}\right)^{2}+\frac{w(r) \lambda}{r^{2} k(r) h(r) \beta^{2}},
\end{aligned}
$$

and $\lambda$ is a separation constant. Here we only consider the radial equation.

Subsequently, as in [34-39], we introduce the generalized tortoise coordinate transformation

$$
r_{*}=r+\frac{1}{2 \kappa} \ln \left(r-r_{+}\right),
$$

which was proposed in $[34,35]$ and extended to study Hawking radiation in dynamical black holes [37-39]. It is interesting that the generalized tortoise coordinate transformation only relies on a parameter $\kappa$ and the location of the horizon. In this paper we will extend this tortoise coordinate transformation to investigate Hawking radiation in fivedimensional charged rotating Kaluza-Klein black holes with squashed horizons. Then using the coordinate transformation (17), we have

$$
\begin{aligned}
& d r_{*}=\left[1+\frac{1}{2 \kappa\left(r-r_{+}\right)}\right] d r, \\
& \frac{d}{d r}=\left[1+\frac{1}{2 \kappa\left(r-r_{+}\right)}\right] \frac{d}{d r_{*}}, \\
& \frac{d^{2}}{d r^{2}}=\left[1+\frac{1}{2 \kappa\left(r-r_{+}\right)}\right]^{2} \frac{d^{2}}{d r_{*}^{2}}-\frac{1}{2 \kappa\left(r-r_{+}\right)^{2}} \frac{d}{d r_{*}} .
\end{aligned}
$$

Then substituting (18) into (14), the radial function in tortoise coordinate system can be written as

$$
\rho_{*}^{2} \frac{d^{2}}{d r_{*}^{2}} R\left(r_{*}\right)+L_{*} \frac{d}{d r_{*}} R\left(r_{*}\right)+R_{*} R\left(r_{*}\right)=0,
$$


where

$$
\begin{aligned}
\rho_{*} & =\frac{w(r)}{\beta k(r) \sqrt{h(r)}}\left[1+\frac{1}{2 \kappa\left(r-r_{+}\right)}\right], \\
L_{*} & =\left[1+\frac{1}{2 \kappa\left(r-r_{+}\right)}\right] B(r)-\frac{w^{2}(r)}{h(r) k^{2}(r) \beta^{2}} \frac{1}{2 \kappa\left(r-r_{+}\right)^{2}}, \\
R_{*} & =C^{2}(r)-D(r) .
\end{aligned}
$$

Notice the value of $\rho_{*}$ at the horizon $r=r_{+}$, so we make a wise choice such that the constant factor $\kappa=\kappa_{\star}$, which lets

$$
\lim _{r \rightarrow r_{+}} \rho_{*}=\lim _{r \rightarrow r_{+}} \frac{w(r)}{\beta k(r) \sqrt{h(r)}}\left[1+\frac{1}{2 \kappa_{\star}\left(r-r_{+}\right)}\right]=1 .
$$

Therefore, it is easy to prove that $r=r_{+}$will lead to

$$
\begin{aligned}
& \lim _{r \rightarrow r_{+}} L_{*}=\lim _{r \rightarrow r_{+}}\left[1+\frac{1}{2 \kappa\left(r-r_{+}\right)}\right] B(r)-\frac{w^{2}(r)}{h(r) k^{2}(r) \beta^{2}} \frac{1}{2 \kappa\left(r-r_{+}\right)^{2}}=0, \\
& \lim _{r \rightarrow r_{+}} R_{*}=\left(E+\frac{f\left(r_{+}\right) L}{\beta}+\frac{\sqrt{3} q e}{2 \beta r_{+}^{2}}\left(1+\frac{a f(r)}{2}\right)\right)^{2},
\end{aligned}
$$

where we have used the equation (16). So, near the event horizon we can simplify the Klein-Gordon equation and get a standard wave equation as follows

$$
\frac{d^{2}}{d r_{*}^{2}} R\left(r_{*}\right)+\left(E-\left(L \Omega+e V_{0}\right)\right)^{2}=0,
$$

where the parameters $\Omega$ and $V_{0}$, which can be seen as the angular velocity and the static electro-potential of the event horizon respectively, are given by

$$
\begin{aligned}
& \Omega=-\frac{f\left(r_{+}\right)}{\beta}, \\
& V_{0}=-\frac{\sqrt{3} q}{2 \beta r_{+}^{2}}\left(1+\frac{a f\left(r_{+}\right)}{2}\right)=-A_{t}+\frac{f\left(r_{+}\right)}{\beta} A_{\varphi} .
\end{aligned}
$$

Two linearly independent solutions are

$$
R\left(r_{*}\right)=e^{ \pm i\left(E-E_{0}\right) r_{*}},
$$

where $E_{0}=L \Omega+e V_{0}$. Therefore, the radial solution can be written as

$$
R_{E}=e^{-i E \tilde{t} \pm i\left(E-E_{0}\right) r_{*}},
$$

where "+" corresponds to an outgoing wave and "-" represents an ingoing wave.

Letting $\hat{r}=\frac{E-E_{0}}{E} r_{*}$, then the radial solution becomes

$$
R_{E}=e^{-i E(\tilde{t} \mp \hat{r})},
$$

Using an advanced Eddington coordinate $v=\tilde{t}+\hat{r}$, in which the metric is well behaved and analytic across the event horizon $r_{+}$, the ingoing and outgoing waves can be expressed separately as

$$
\begin{gathered}
R_{E}^{i n}=e^{-i E(\tilde{t}+\hat{r})}=e^{-i E v}, \\
R_{E}^{\text {out }}=e^{-i E(\tilde{t}-\hat{r})}=e^{-i E v} \cdot e^{2 i E \hat{r}}=e^{-i E v} \cdot e^{2 i\left(E-E_{0}\right) r_{*}} .
\end{gathered}
$$

Notice that (26) is divergent on the horizon $r=r_{+}$, and the outgoing waves cannot be extended straightforwardly to the region inside the horizon. Therefore, we need to extend the 
outgoing wave equation (26) into the horizon by turning $(-\pi)$ angle through the negative half complex plane. Letting

$$
\left(r-r_{+}\right) \rightarrow\left|r-r_{+}\right| e^{-i \pi}=\left(r_{+}-r\right) e^{-i \pi},
$$

the outgoing wave function inside and outside of the horizon are

$$
\begin{gathered}
R_{E}^{\text {out }}\left(r<r_{+}\right)=e^{-i E v}\left(r_{+}-r\right)^{\frac{i}{\kappa_{\star}}\left(E-E_{0}\right)} e^{\frac{\pi}{\kappa_{\star}}\left(E-E_{0}\right)}, \\
R_{E}^{\text {out }}\left(r>r_{+}\right)=e^{-i E v}\left(r-r_{+}\right)^{\frac{i}{\kappa_{\star}}}\left(E-E_{0}\right)
\end{gathered}
$$

According to Damour-Sannan's work [2], the emission rate at the horizon can be obtained as

$$
\Gamma=\left|\frac{R_{E}^{\text {out }}\left(r>r_{+}\right)}{R_{E}^{\text {out }}\left(r<r_{+}\right)}\right|^{2}=e^{-\frac{2 \pi}{\kappa_{\star}}\left(E-E_{0}\right)} .
$$

Then it is easy to get the thermal radiation spectrum

$$
N_{E}=\frac{1}{e^{\frac{E-E_{0}}{k_{B} T}} \pm 1}
$$

where

$$
T=\frac{\kappa_{\star}}{2 \pi k_{B}}=\frac{r_{+}^{2}-r_{-}^{2}}{\pi k_{B}\left(r_{\infty}^{2}-r_{-}^{2}\right)} \sqrt{\frac{r_{\infty}^{2}-r_{+}^{2}}{r_{\infty}^{2}-r_{-}^{2}}} \frac{r_{\infty} l}{\sqrt{r_{+}^{6}-a^{2} q^{2}+2 a^{2}(m+q) r_{+}^{2}}}
$$

in which "+" corresponds to fermions and "-" represents bosons, $k_{B}$ is the Boltzmann constant, $\kappa_{\star}, T$ are surface gravity and Hawking temperature, respectively. This conclusion is consistent with the previous result in [23]. It is obvious that Hawking temperature is proportional to the surface gravity and related to the size of extra dimension in the squashed charged rotating Kaluza-Klein black hole.

\section{Hawking Radiation of Charged Dirac Fermions}

In this section, we intend to calculate the thermal spectrum of the squashed charged rotating Kaluza-Klein black hole due to the Hawking radiation of Dirac particles. We start by writing the Dirac equation for an electrically charged particle in covariant coordinate system,

$$
i \gamma^{\mu}\left(D_{\mu}-\frac{i e}{\hbar} A_{\mu}\right) \psi+\frac{\tilde{m}}{\hbar} \psi=0 .
$$

Here $e$ and $\tilde{m}$ denote charge and mass of the emitted fermion particle, respectively, $A_{\mu}$ is the 4-potential, $\psi$ is the wave function and

$$
D_{\mu}=\partial_{\mu}+\Gamma_{\mu}, \Gamma_{\mu}=\frac{1}{8}\left[\gamma^{a}, \gamma^{b}\right] e_{a}^{\nu e_{b v ; \mu}}, e_{b v ; \mu}=\partial_{\mu e_{b v}}-\Gamma_{\mu \nu}^{\alpha} e_{b \alpha},
$$


where $\Gamma_{\mu \nu}^{\alpha}$ is the Christoffel symbol, and $e_{a}^{v}$ is the tetrad given by

$$
e_{a}^{v}=\left(\begin{array}{ccccc}
\beta \sqrt{\frac{h(r)}{w(r)}} & 0 & 0 & 0 & -f(r) \sqrt{\frac{h(r)}{w(r)}} \\
0 & 0 & \frac{2}{r \sqrt{k(r)}} & 0 & 0 \\
0 & 0 & 0 & \frac{2}{r \sin \theta \sqrt{k(r)}} & -\frac{2 \cot \theta}{r \sqrt{k(r)}} \\
0 & \frac{\sqrt{w(r)}}{k(r)} & 0 & 0 & 0 \\
0 & 0 & 0 & 0 & \frac{2}{r \sqrt{h(r)}}
\end{array}\right)
$$

The inverse of the tetrad $e_{v}^{a}$ is defined by

$$
g_{\mu v}=\eta_{a b} e_{\mu}^{a} e_{v}^{b}
$$

with $\eta^{a b}=\operatorname{diag}(-1,1,1,1,1)$, the Minkowski metric. The $\gamma^{\mu}$ matrices satisfy the communication relation $\left\{\gamma^{\mu}, \gamma^{\nu}\right\}=2 g^{\mu \nu} \hat{I}$. As in [23], we choose the matrices $\gamma^{\mu}$ as

$$
\begin{aligned}
\gamma^{\tilde{t}} & =\beta \sqrt{\frac{h(r)}{w(r)}} \gamma^{0}, \gamma^{r}=\frac{\sqrt{w(r)}}{k(r)} \gamma^{3}, \\
\gamma^{\theta} & =\frac{2}{r \sqrt{k(r)}} \gamma^{1}, \gamma^{\phi}=\frac{2}{r \sqrt{k(r)} \sin \theta} \gamma^{2}, \\
\gamma^{\varphi} & =-f(r) \sqrt{\frac{h(r)}{w(r)}} \gamma^{0}-\frac{2 \cot \theta}{r \sqrt{k(r)}} \gamma^{2}+\frac{2}{r \sqrt{h(r)}} \gamma^{4} .
\end{aligned}
$$

and the matrices $\gamma^{a}$ take the form

$$
\begin{aligned}
& \gamma^{0}=\left(\begin{array}{cc}
0 & I \\
-I & 0
\end{array}\right), \gamma^{1}=\left(\begin{array}{cc}
0 & \sigma_{1} \\
\sigma_{1} & 0
\end{array}\right), \\
& \gamma^{2}=\left(\begin{array}{cc}
0 & \sigma_{2} \\
\sigma_{2} & 0
\end{array}\right), \gamma^{3}=\left(\begin{array}{cc}
0 & \sigma_{3} \\
\sigma_{3} & 0
\end{array}\right), \gamma^{4}=\left(\begin{array}{cc}
-I & 0 \\
0 & I
\end{array}\right),
\end{aligned}
$$

where the $\sigma_{i}$ are simply the Pauli Sigma matrices

$$
\sigma_{1}=\left(\begin{array}{ll}
0 & 1 \\
1 & 0
\end{array}\right), \sigma_{2}=\left(\begin{array}{cc}
0 & -i \\
-i & 0
\end{array}\right), \sigma_{3}=\left(\begin{array}{cc}
1 & 0 \\
0 & -1
\end{array}\right) .
$$

Then using the metric (1), one can obtain the spin connection $\Gamma_{\mu}$ as

$$
\begin{aligned}
\Gamma_{0} & =\frac{\gamma^{1} \cdot \gamma^{2} h(r) f(r)}{4 \beta k(r)}-\frac{\gamma^{3} \cdot \gamma^{4} \sqrt{w(r)}}{8 \beta k(r) \sqrt{h(r)}}\left[2 f(r) h(r)+r h(r) f^{\prime}(r)+r f(r) h^{\prime}(r)\right] \\
& +\frac{\gamma^{0} \cdot \gamma^{3} r^{2} f(r) h(r)^{\frac{3}{2}} f^{\prime}(r)}{16 \beta k(r)}+\frac{\gamma^{0} \cdot \gamma^{3} \sqrt{h(r)} w(r) h^{\prime}(r)}{4 \beta h^{2}(r) k(r)}-\frac{\gamma^{0} \cdot \gamma^{3} \sqrt{h(r)} w^{\prime}(r)}{4 \beta h(r) k(r)}, \\
\Gamma_{1} & =\frac{5 \gamma^{0} \cdot \gamma^{4} r^{4} h(r) f^{\prime}(r)}{8 \sqrt{w(r)}}, \\
\Gamma_{2} & =\frac{\gamma^{1} \cdot \gamma^{3} \sqrt{w(r)}}{8 k(r)^{\frac{3}{2}}}\left[2 k(r)+r k^{\prime}(r)\right], \\
\Gamma_{3} & =\frac{\gamma^{0} \cdot \gamma^{3} r^{2} \cos \theta h^{2}(r) f^{\prime}(r)}{16 \sqrt{h(r)} k(r)}+\frac{\gamma^{1} \cdot \gamma^{2} \cos \theta}{4 k(r)}[h(r)-2 k(r)]+\frac{\gamma^{1} \cdot \gamma^{4} \sin \theta \sqrt{h(r)}}{4 \sqrt{k(r)}} \\
& -\frac{\gamma^{3} \cdot \gamma^{4} \cos \theta \sqrt{w(r)}}{8 \sqrt{h(r)} k(r)}\left[2 h(r)+r h^{\prime}(r)\right]+\frac{\gamma^{2} \cdot \gamma^{3} \sin \theta \sqrt{w(r)}}{8 k(r)^{\frac{3}{2}}}\left[2 k(r)+r k^{\prime}(r)\right], \\
\Gamma_{4} & =\frac{\gamma^{0} \cdot \gamma^{3} r^{2} h^{2}(r) f^{\prime}(r)}{16 \sqrt{h(r)} k(r)}+\frac{\gamma^{1} \cdot \gamma^{2} h(r)}{4 k(r)}-\frac{\gamma^{3} \cdot \gamma^{4} \sqrt{w(r)}}{8 \sqrt{h(r)} k(r)}\left[2 h(r)+r h^{\prime}(r)\right] .
\end{aligned}
$$


According to the symmetry of the squashed charged rotating Kaluza-Klein black hole, we employ an ansatz for the Dirac spinor as

$$
\psi(t, r, \theta, \phi, \varphi)=\left(\begin{array}{l}
f_{1}(r, \theta) \\
f_{2}(r, \theta) \\
g_{1}(r, \theta) \\
g_{2}(r, \theta)
\end{array}\right) \mathrm{e}^{-i(E \tilde{t}-J \phi-L \varphi)} .
$$

Then substituting (33), (34), (37)and (38) into (32), the Dirac equation can be written as

$$
\begin{aligned}
& {\left[r \beta \sqrt{\frac{h(r) k(r)}{w(r)}} I(r)+i\left(F(r)+r \sqrt{\frac{w(r)}{k(r)}} \partial_{r}\right)\right] g_{1}(r, \theta)} \\
& +\left(2 i \partial_{\theta}+\frac{2 i J}{\sin \theta}-2 i L \cot \theta\right) g_{2}(r, \theta)+\sqrt{\frac{k(r)}{h(r)}}(G(r)+H(r)) f_{1}(r, \theta)=0, \\
& {\left[r \beta \sqrt{\frac{h(r) k(r)}{w(r)}} I(r)-i\left(F(r)+r \sqrt{\frac{w(r)}{k(r)}} \partial_{r}\right)\right] g_{2}(r, \theta)} \\
& +\left(2 i \partial_{\theta}-\frac{2 i J}{\sin \theta}+2 i L \cot \theta\right) g_{1}(r, \theta)+\sqrt{\frac{k(r)}{h(r)}}(G(r)-H(r)) f_{2}(r, \theta)=0, \\
& \quad+\left(2 i \partial_{\theta}+\frac{2 i J}{\sin \theta}-2 i L \cot \theta\right) f_{2}(r, \theta)+\sqrt{\frac{k(r)}{h(r)}}(M(r)+N(r)) g_{1}(r, \theta)=0, \\
& \left.\quad r \beta \sqrt{\frac{h(r) k(r)}{w(r)}} I(r)+i\left(F(r)+r \sqrt{\frac{w(r)}{k(r)}} \partial_{r}\right)\right] f_{1}(r, \theta) \\
& \quad+\left(2 i \partial_{\theta}-\frac{2 i J}{\sin \theta}+2 i L \cot \theta\right) f_{1}(r, \theta)+\sqrt{\frac{k(r)}{h(r)}}(M(r)-N(r)) g_{2}(r, \theta)=0,
\end{aligned}
$$

where

$$
\begin{aligned}
& I(r)=E+\frac{f(r) L}{\beta}+\frac{\sqrt{3} q e}{2 \beta r^{2}}\left(1+\frac{a f(r)}{2}\right) \\
& F(r)=\frac{r w^{\prime}(r)}{4 \sqrt{k(r) w(r)}}+\frac{3}{2} \sqrt{\frac{w(r)}{k(r)}}+\frac{r \sqrt{w(r) k(r)} k^{\prime}(r)}{2 k^{2}(r)} \\
& G(r)=2 L+\frac{\sqrt{3} q e a}{2 r^{2}}+r \sqrt{h(r)} \tilde{m} \\
& H(r)=\frac{5 i r^{2} h(r) \sqrt{h(r)} f^{\prime}(r)}{8 k(r)}-\frac{5 h(r)}{2 k(r)} \\
& M(r)=-2 L-\frac{\sqrt{3} q e a}{2 r^{2}}+r \sqrt{h(r)} \tilde{m}, \\
& N(r)=\frac{5 i r^{2} h(r) \sqrt{h(r)} f^{\prime}(r)}{8 k(r)}+\frac{5 h(r)}{2 k(r)}
\end{aligned}
$$


Now we could separate the variables as follows

$$
\begin{aligned}
& f_{1}(r, \theta)=R_{-\frac{1}{2}}(r) S_{-\frac{1}{2}}(\theta), \\
& f_{2}(r, \theta)=R_{+\frac{1}{2}}(r) S_{+\frac{1}{2}}(\theta), \\
& g_{1}(r, \theta)=R_{+\frac{1}{2}}(r) S_{-\frac{1}{2}}(\theta), \\
& g_{2}(r, \theta)=R_{-\frac{1}{2}}(r) S_{+\frac{1}{2}}(\theta) .
\end{aligned}
$$

Then substituting (43) into (39) and (40), we can get the radial equation

$$
\begin{gathered}
{\left[r \beta \sqrt{\frac{h(r) k(r)}{w(r)}} I(r)+i\left(F(r)+r \sqrt{\frac{w(r)}{k(r)}} \frac{d}{d r}\right)\right] R_{+\frac{1}{2}}(r)} \\
=\left[\lambda-\sqrt{\frac{k(r)}{h(r)}} G(r)-\sqrt{\frac{k(r)}{h(r)}} H(r)\right] R_{-\frac{1}{2}}(r), \\
{\left[r \beta \sqrt{\frac{h(r) k(r)}{w(r)}} I(r)-i\left(F(r)+r \sqrt{\frac{w(r)}{k(r)}} \frac{d}{d r}\right)\right] R_{-\frac{1}{2}}(r)} \\
=\left[\lambda-\sqrt{\frac{k(r)}{h(r)}} G(r)+\sqrt{\frac{k(r)}{h(r)}} H(r)\right] R_{+\frac{1}{2}}(r),
\end{gathered}
$$

and the angular equation

$$
\begin{aligned}
& \left(2 i \frac{d}{d \theta}+\frac{2 i J}{\sin \theta}-2 i L \cot \theta\right) S_{+\frac{1}{2}}(\theta)+\lambda S_{-\frac{1}{2}}(\theta)=0 \\
& \left(2 i \frac{d}{d \theta}-\frac{2 i J}{\sin \theta}+2 i L \cot \theta\right) S_{-\frac{1}{2}}(\theta)+\lambda S_{+\frac{1}{2}}(\theta)=0
\end{aligned}
$$

respectively. Using the equations (44) and (45), one can get the radial equation $R_{-\frac{1}{2}}(r)$

$$
\frac{w^{2}(r)}{\beta^{2} h(r) k^{2}(r)} \frac{d^{2}}{d r^{2}} R_{-\frac{1}{2}}(r)+O(r) \frac{d}{d r} R_{-\frac{1}{2}}(r)+P(r) R_{-\frac{1}{2}}(r)=0,
$$

where

$$
\begin{aligned}
O(r)= & \frac{w(r) \sqrt{w(r)} F(r)}{r \beta^{2} h(r) k(r) \sqrt{k(r)}}+\frac{w^{2}(r)}{r \beta^{2} h(r) k^{2}(r)}+\frac{w(r) w^{\prime}(r)}{2 \beta^{2} h(r) k^{2}(r)}-\frac{w^{2}(r) k^{\prime}(r)}{2 \beta^{2} h(r) k^{3}(r)} \\
& +\frac{w^{2}(r)}{\beta^{2} h(r) k^{2}(r)} \frac{\left[\lambda-\sqrt{\frac{k(r)}{h(r)}}(G(r)-H(r))\right]^{\prime}}{\lambda-\sqrt{\frac{k(r)}{h(r)}}(G(r)-H(r))},
\end{aligned}
$$




$$
\begin{aligned}
P(r)= & I^{2}(r)+\frac{w(r)}{r^{2} \beta^{2} h(r) k(r)} B^{2}(r)+\frac{w(r) \sqrt{w(r)} F^{\prime}(r)}{r \beta^{2} h(r) k(r) \sqrt{k(r)}} \\
& +\frac{i w(r) \sqrt{w(r)}}{r \beta^{2} h(r) k(r) \sqrt{k(r)}}\left[r \beta \sqrt{\frac{h(r) k(r)}{w(r)}} I(r)\right]^{\prime} \\
& -\frac{\left[\lambda-\sqrt{\frac{k(r)}{h(r)}}(G(r)-H(r))\right]^{\prime}}{\lambda-\sqrt{\frac{k(r)}{h(r)}}(G(r)-H(r))}\left[\frac{w(r) \sqrt{h(r)}}{\beta h(r) k(r)} I(r)+\frac{w(r) \sqrt{w(r)}}{r \beta^{2} h(r) k(r) \sqrt{k(r)}} F(r)\right] \\
& -\frac{w(r)}{r^{2} \beta^{2} h(r) k(r)}\left[\left(\lambda-\sqrt{\frac{k(r)}{h(r)}} G(r)\right)^{2}-H^{2}(r)\right] .
\end{aligned}
$$

and the angular equation $S_{-\frac{1}{2}}(\theta)$

$$
\frac{d^{2}}{d \theta^{2}} S_{-\frac{1}{2}}(\theta)+\frac{1}{\sin ^{2} \theta}(\cos \theta J-L) S_{-\frac{1}{2}}(\theta)-\frac{1}{\sin ^{2} \theta}(J-L \cos \theta)^{2} S_{-\frac{1}{2}}(\theta)+\frac{\lambda^{2}}{4} S_{-\frac{1}{2}}(\theta)=0 .
$$

Similarly, we can obtain the equations of $R_{+\frac{1}{2}}(r)$ and $S_{+\frac{1}{2}}(\theta)$.

Subsequently, we only consider the radial equation (46). Letting $R_{-\frac{1}{2}}(r)=R(r)$, under the generalized tortoise coordinate transformation (17), the radial function (46) can be written as

$$
\eta_{*}^{2} \frac{d^{2}}{d r_{*}^{2}} R\left(r_{*}\right)+M_{*} \frac{d}{d r_{*}} R\left(r_{*}\right)+N_{*} R\left(r_{*}\right)=0,
$$

where

$$
\begin{aligned}
\eta_{*} & =\frac{w(r)}{\beta k(r) \sqrt{h(r)}}\left[1+\frac{1}{2 \kappa\left(r-r_{+}\right)}\right], \\
M_{*} & =\left[1+\frac{1}{2 \kappa\left(r-r_{+}\right)}\right] O(r)-\frac{w^{2}(r)}{\beta^{2} h(r) k^{2}(r)} \frac{1}{2 \kappa\left(r-r_{+}\right)^{2}}, \\
N_{*} & =P(r) .
\end{aligned}
$$

We make a wise choice such that the constant factor $\kappa=\kappa_{\star}$, which lets

$$
\lim _{r \rightarrow r_{+}} \eta_{*}=\lim _{r \rightarrow r_{+}} \frac{w(r)}{\beta k(r) \sqrt{h(r)}}\left[1+\frac{1}{2 \kappa_{\star}\left(r-r_{+}\right)}\right]=1 .
$$

Substituting $\kappa_{\star}$ into $M_{*}$ and $N_{*}$, then

$$
\begin{aligned}
& \lim _{r \rightarrow r_{+}} M_{*}=\lim _{r \rightarrow r_{+}}\left[1+\frac{1}{2 \kappa_{\star}\left(r-r_{+}\right)}\right] O(r)-\frac{w^{2}(r)}{\beta^{2} h(r) k^{2}(r)} \frac{1}{2 \kappa_{\star}\left(r-r_{+}\right)^{2}}=\frac{1}{4 \kappa_{\star} \beta^{2}} \frac{\left[w^{\prime}\left(r_{+}\right)\right]^{2}}{h\left(r_{+}\right) k^{2}\left(r_{+}\right)}, \\
& \lim _{r \rightarrow r_{+}} N_{*}=\lim _{r \rightarrow r_{+}} P(r)=I^{2}\left(r_{+}\right)-\frac{i w^{\prime}\left(r_{+}\right)}{2 \beta k\left(r_{+}\right) \sqrt{h\left(r_{+}\right)}} I\left(r_{+}\right) .
\end{aligned}
$$


When $r \rightarrow r_{+}$, substituting (51) and (52) into (49), this leads to the desired result

$$
\frac{d^{2}}{d r_{*}^{2}} R\left(r_{*}\right)+\frac{1}{4 \kappa_{\star} \beta^{2}} \frac{\left[w^{\prime}\left(r_{+}\right)\right]^{2}}{h\left(r_{+}\right) k^{2}\left(r_{+}\right)} \frac{d}{d r_{*}} R\left(r_{*}\right)+\left(I^{2}\left(r_{+}\right)-\frac{i \sqrt{h\left(r_{+}\right)} w^{\prime}\left(r_{+}\right)}{2 \beta h\left(r_{+}\right) k\left(r_{+}\right)} I\left(r_{+}\right)\right) R\left(r_{*}\right)=0,
$$

which is a wave equation. It is easy to get the solution

$$
R \sim\left\{\begin{array}{c}
e^{i\left(E-E_{0}\right) r_{*}}, \\
0 .
\end{array}\right.
$$

It is obvious that the non-zero solution is

$$
R \sim e^{i\left(E-E_{0}\right) r_{*}},
$$

So the radial wave function can be written as

$$
R_{E}=e^{-i E \tilde{t}+i\left(E-E_{0}\right) r_{*}}=e^{-i E\left(\tilde{t}-\frac{E-E_{0}}{E} r_{*}\right)}=e^{-i E(\tilde{t}-\hat{r})},
$$

where $\hat{r}=\frac{E-E_{0}}{E} r_{*}$. One can find that the equation (54) describes the outgoing wave from the horizon

$$
R_{E}^{\text {out }} \sim e^{-i E(\tilde{t}-\hat{r})} .
$$

Using an advanced Eddington coordinate $v=\tilde{t}+\hat{r}$, we can get the equation of outgoing wave

$$
R_{E}^{\text {out }} \sim e^{-i E v+i 2\left(E-E_{0}\right) r_{*}}=e^{-i E v} e^{i \frac{E-E_{0}}{\kappa_{\star}} \ln \left(r-r_{+}\right)}=e^{-i E v}\left(r-r_{+}\right)^{i \frac{E-E_{0}}{\kappa_{\star}}} .
$$

Now it is obvious that the outgoing wave function can only describe the outgoing particles behavior outside the horizon. In order to extend the outgoing wave function inside the horizon, letting $\left(r-r_{+}\right) \rightarrow\left|r-r_{+}\right| e^{-i \pi}=\left(r_{+}-r\right) e^{-i \pi}$, the outgoing wave function inside the horizon can be obtained as

$$
R_{E}^{\text {out }}\left(r<r_{+}\right)=e^{-i E v}\left(r_{+}-r\right)^{\frac{i}{\kappa_{\star}}\left(E-E_{0}\right)} e^{\frac{\pi}{\kappa_{\star}}\left(E-E_{0}\right)} .
$$

So we can write the total outgoing wave function as

$$
\begin{aligned}
& R_{E}^{\text {out }}\left(r<r_{+}\right)=e^{-i E v}\left(r_{+}-r\right)^{\frac{i}{\kappa_{\star}}\left(E-E_{0}\right)} e^{\frac{\pi}{\kappa_{\star}}\left(E-E_{0}\right)}, \\
& R_{E}^{\text {out }}\left(r>r_{+}\right)=e^{-i E v}\left(r-r_{+}\right)^{\frac{i}{\kappa_{\star}}\left(E-E_{0}\right)} .
\end{aligned}
$$

Therefore, according to Damour-sannan's work [2], the emission rate at the horizon can be given

$$
\Gamma=\left|\frac{R_{E}^{\text {out }}\left(r>r_{+}\right)}{R_{E}^{\text {out }}\left(r<r_{+}\right)}\right|^{2}=e^{-\frac{2 \pi}{k_{\star}}\left(E-E_{0}\right)}
$$

and the corresponding thermal temperature is

$$
T=\frac{\kappa_{\star}}{2 \pi k_{B}}=\frac{r_{+}^{2}-r_{-}^{2}}{\pi k_{B}\left(r_{\infty}^{2}-r_{-}^{2}\right)} \sqrt{\frac{r_{\infty}^{2}-r_{+}^{2}}{r_{\infty}^{2}-r_{-}^{2}}} \frac{r_{\infty} l}{\sqrt{r_{+}^{6}-a^{2} q^{2}+2 a^{2}(m+q) r_{+}^{2}}}
$$

which is the same as the Hawking temperature experienced by the charged scalar particles. Therefore we could conclude that the Hawking temperature from the five-dimensional 
squashed charged rotating Kaluza-Klein black hole is independent of the properties of particles in our approach.

\section{Conclusions}

In the present paper, we have calculated Hawking radiation of charged particles using the Damour-Ruffini method from the five-dimensional squashed charged rotating KaluzaKlein black holes. After introducing the generalized tortoise coordinate transformation, we find that the radial motion equations of emission particles can be simplified into the wave equations near the horizon. Then the Hawking temperature can be obtained using the corresponding emission rate in the tunneling mechanism. Thus we successfully extend the Damour-Ruffini method to discuss the Hawking radiation in high dimensional black holes with the nontrivial topology horizon. What's more, our results also show that Hawking temperature for charged Dirac fermions is the same as for charged scalar particles. That is to say Hawking temperature from the five-dimensional squashed charged rotating Kaluza-Klein black hole are independent of the properties of particles in our approach. Finally, from the equations (30) and 31, one can find that the thermal spectrum of radiation contain the information of the size of the extra dimension, which could open a possible window to observe extra dimensions in the future.

Acknowledgments This research is supported by the National Natural Science Foundation of China (Grant Nos. 11365008, 51278512). Xian-Ming Liu is also supported by and the Natural Science Foundation of Hubei Province (Grant No. 2014CFB608). Jing-He Wu is also supported by the Key Scientific and Technological Project of Henan Province (Grant No.152102210307) and the Natural Science Foundation of Henan Educational Committee (Grant No.2011A140006).

Open Access This article is distributed under the terms of the Creative Commons Attribution 4.0 International License (http://creativecommons.org/licenses/by/4.0/), which permits unrestricted use, distribution, and reproduction in any medium, provided you give appropriate credit to the original author(s) and the source, provide a link to the Creative Commons license, and indicate if changes were made.

\section{References}

1. Damour, T., Ruffini, R.: Phys Rev. D 14, 332 (1976)

2. Sannan, S.: Gen. Rel. Grav. 20, 239 (1988)

3. Hawking, S.W.: Nature 248, 30 (1974)

4. Hawking, S.W.: Commun. Math. Phys. 43, 199 (1975)

5. Parikh, M.K., Wilczek, F.: Phys. Rev. Lett. 85, 5042 (2000)

6. Parikh, M.K.: Int. J. Mod. Phys. D 13, 2351 (2004)

7. Parikh, M.K.: Energy conservation and Hawking radiation. arXiv:hep-th/0402166 (2004)

8. Zhang, J.Y., Zhao, Z.: Phys. Lett. B 638, 110 (2006)

9. Srinivasan, K., Padmanabhan, T.: Phys. Rev. D 60, 024007 (1999)

10. Shankaranarayanan, S., Padmanabhan, T., Srinivasan, K.: Class. Quantum Gravity 19, 2671 (2002)

11. Angheben, M., Nadalini, M., Vanzo, L., Zerbini, S.: J. High Energy Phys. 2005, 014 (2005)

12. Kerne, R., Mann, R.B.: Phys. Rev. D 73, 104010 (2006)

13. Ishihara, H., Matsuno, K.: Prog. Theor. Phys. 116, 417 (2006)

14. Yazadjiev, S.S.: Phys. Rev. D 74, 024022 (2006)

15. Brihaye, Y., Radu, E.: Phys. Lett. B 641, 212 (2006)

16. Ishihara, H., Kimura, M., Matsuno, K., Tomizawa, S.: Phys. Rev. D 74, 047501 (2006)

17. Ishihara, H., Kimura M., Matsuno, K., Tomizawa, S.: Class. Quant. Grav. 6919, 23 (2006)

18. Harmark, T., Niarchos, V., Andobers, N.A.: Class Quant. Grav. 24, R1 (2007) 
19. Wang, T.: Nucl. Phys. B 756, 86 (2006)

20. Tomizawa, S., Ishihara, H., Matsuno, K., Nakagawa, T.: Prog. Theor. Phys. 121, 823 (2009)

21. Ishihara, H., Soda, J.: Phys. Rev. D 76, 064022 (2007)

22. Chen, S., Wang, B., Su, R.-K.: Phys. Rev. D 77, 024039 (2008)

23. Stetskoa, M.M.: Eur. Phys. J. C 76, 48 (2016)

24. He, X., Wang, B., Chen, S., Cai, R.-G., Lin, C.-Y.: Phys. Lett. B 665, 392 (2008)

25. He, X., Wang, B., Chen, S.: Phys. Rev. D 79, 084005 (2009)

26. Ishihara, H. et al.: Phys. Rev. D 77, 084019 (2008)

27. Matsuno, K., Ishihara, H.: Phys. Rev. D 80, 104037 (2009)

28. Peng, J., Wu, S.: Nucl.Phys.B 828, 273 (2010)

29. Chen, S., Liu, Y., Jing, J.: Phys. Rev. D 83, 124019 (2011)

30. Ji, L., Chen, S., Jing, J.: JHEP 03, 089 (2014)

31. Cvetic, M., Youm, D.: Nucl. Phys. B476, 118 (1996)

32. Cvetic, M., Lu, H., Pope, C.N.: Phys. Lett. B598, 273 (2004)

33. Nakagawa, T., Ishihara, H., Matsuno, K., Tomizawa, S.: Phys. Rev. D77, 044040 (2008)

34. Zhao, Z., Gui, Y.X., Liu, L.: Chin. Astron. Astrophys. 1, 141 (1981)

35. Zhao, Z., Gui, Y.X.: Chin. Astron. Astrophys. 3, 146 (1983)

36. Zhou, S., Liu, W.: Phys. Rev. D 77, 104021 (2008)

37. Zhao, Z., Dai, X.: Chin. Phys. Lett. 8, 548 (1991)

38. Liu, X., Liu, W.: Astrophys Space Sci. 331, 237 (2011)

39. Liu, X., Cheng, S., Liu, W.: Int J Theor Phys. 51, 518 (2012) 